\title{
Distinct Functional Types of Associative Long-Term Potentiation in Neocortical and Hippocampal Pyramidal Neurons
}

\author{
Dean V. Buonomano \\ Departments of Neurobiology and Psychology, and Brain Research Institute, University of California, Los Angeles, \\ Los Angeles, California 90095
}

The response of a neuron to a time-varying stimulus is influenced by both short- and long-term synaptic plasticity. Both these forms of plasticity produce changes in synaptic efficacy of similar magnitude on very different time scales. A full understanding of the functional role of each form of plasticity relies on understanding how they interact. Here we examine how longterm potentiation (LTP) and short-term plasticity (STP) interact in two different cell types that exhibit NMDA-dependent LTP: neocortical L-II/III and hippocampal CA1 pyramidal cells. STP was examined using both paired pulses and trains of pulses before and after the induction of LTP. In both cell types, the same pairing protocol was used to induce LTP in the presence of an unpaired control pathway. Pairing produced a robust increase in the amplitude of the first EPSP both in the neocortex and hippocampus. However, although in CA1 neurons the same degree of potentiation was maintained throughout the duration of a brief stimulus train, in L-II/III neurons relatively less potentiation was seen in the later EPSPs of the train. Paired-pulse analyses revealed that a uniform potentiation is observed at intervals $>100 \mathrm{msec}$, but at shorter intervals there is a preferential enhancement of the first pulse. Thus, in the cortex LTP may preferentially amplify stimulus onset. These results suggest that there are distinct forms of associative LTP and that the different forms may reflect the underlying computations taking place in different areas.

Key words: long-term potentiation; short-term potentiation; associative; hippocampus; neocortex; pyramidal
Long-term changes in synaptic efficacy have been proposed to underlie various forms of learning and of cortical reorganization. In general, these proposals assume that a form of associative long-term potentiation (LTP) produces an increase in the gain of a synapse. That is, after LTP all activity flowing through that synapse is amplified. However, research on neocortical LTP between L-V pyramidal neurons, using trains of activity, has shown that although LTP results in the potentiation of the first EPSP of a train, little or no potentiation of the latter EPSPs may be observed (Markram and Tsodyks, 1996). If such a finding is a general feature of neocortical LTP, it has important implications as to the role of LTP in learning and memory. For example, it is thought that LTP between L-II/III pyramidal neurons in the barrel cortex may underlie experience-dependent changes in receptive fields of these neurons measured by vibrissae stimulation (Armstrong-James et al., 1994; Diamond et al., 1994) as well as other forms of neocortical plasticity (Buonomano and Merzenich, 1998a). If LTP at these synapses were to potentiate only the first EPSP of a series, it would be expected that the expression of the new receptive fields be primarily confined to the first of a series of stimuli.

Whether or not LTP produces a constant change in the gain of a synapse also has important implications for the understanding of the functional role of short-term synaptic plasticity. Short-term plasticity (STP) refers to use-dependent changes in synaptic efficacy occurring on the time scale of tens to hundreds of millisec-

Received March 19, 1999; revised May 21, 1999; accepted May 26, 1999.

This research was supported by the Alfred P. Sloan Foundation. I thank Tom O'Dell for reading an earlier version of this manuscript.

Correspondence should be addressed to Dean V. Buonomano, University of California, Los Angeles, 695 Young Drive South, Room 1320, Box 951761, Los Angeles, CA 90095.

Copyright (C) 1999 Society for Neuroscience 0270-6474/99/196748-07\$05.00/0 onds. Among other hypotheses, short-term plasticity has been proposed to play a role in temporal processing (Buonomano and Merzenich, 1995; Buonomano et al., 1997). If LTP interacts with STP, that is, alters the temporal profile of postsynaptic responses, it would be expected to modify the temporal selectivity of neurons. Under this scenario, LTP would not increase the response throughout the duration of a stimulus, but rather serve to "amplify" stimulus onset. If, on the other hand, LTP does not interact with STP, temporal selectivity is likely to be preserved. Figure 1 schematizes these different instances by showing how, starting from the same initial state, two types of LTP could lead to very different outcomes regarding the temporal response characteristics of a neuron.

The goal of the current paper was to examine the interaction between LTP and STP at both L-II/III auditory cortex pyramidal neurons and at CA1 hippocampal neurons. Brief trains and paired pulses were used as measure of STP, before and after the induction of LTP. By using the same protocol in neocortical and hippocampal experiments, we were able to show two distinct forms of associative LTP in regard to its effect on STP. It is shown that L-II/III pyramidal neurons exhibit a clear interaction between LTP and STP, specifically, EPSPs following the first exhibit relatively less potentiation. Paired-pulse stimulation also revealed a smaller degree of potentiation of the second EPSP of a pair for paired-pulse intervals of 50 and $100 \mathrm{msec}$, but not for intervals of 200 and $300 \mathrm{msec}$, indicating that changes in the temporal profile of the postsynaptic response are limited to a time scale $<200$ msec. In contrast to L-II/III neurons, the same induction protocol induced LTP that was uniform across all EPSPs of a train of stimuli in hippocampal CA1 neurons. Together these results suggest that LTP and STP may have different functional roles at different synapses and that, in biochemical mechanisms, must be 
A.

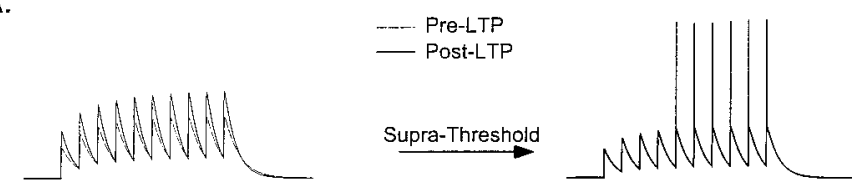

B.

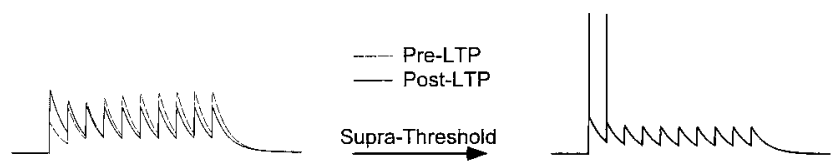

Figure 1. Schematic of how the interaction between LTP and STP can effect neuronal responses to temporal stimuli. If a cell initially exhibits some facilitation in response to a train of inputs, LTP can result in two different scenarios. $A$, If the induction of LTP does not modify the short-term facilitation, the temporal response characteristics remain unchanged. Suprathreshold responses will reflect the facilitation and produce a neuron that selectively responds to prolonged inputs. $B$, If LTP modifies short-term plasticity by producing proportionally larger potentiation of the first EPSP, suprathreshold responses will favor detection of the stimulus onset and not of the later temporal features of a stimulus.

in place to regulate both LTP and STP in an orchestrated manner.

\section{MATERIALS AND METHODS}

Hippocampal and auditory cortex slice experiments were performed on $400-\mu$ m-thick slices from 18- to 30-d-old Sprague Dawley rats. During an equilibration period of at least $1 \mathrm{hr}$, slices were submerged in a oxygenated medium comprised of (in $\mathrm{mm}$ ): $\mathrm{NaCl} 119, \mathrm{KCl} 2.5, \mathrm{MgSO}_{4} 1.3$, $\mathrm{NaH}_{2} \mathrm{PO}_{4} 1.0, \mathrm{NaCO}_{3} 26.2, \mathrm{CaCl}_{2} 2.5$, and glucose 10. Slices were transferred to a submerged recording chamber perfused at a rate of 2 $\mathrm{ml} / \mathrm{min}$ and maintained at a temperature of $30-31^{\circ} \mathrm{C}$.

Auditory cortex experiments. For auditory cortex slices, the brain was removed and cut into two hemispheres. One hemisphere was placed with the medial surface on an agar block, and transverse slices were then cut starting from the posterior end. In the rat, the auditory cortex occupies an area of $\sim 15-20 \mathrm{~mm}^{2}$ of the dorsolateral surface of the temporal lobe (Winer and Larue, 1987; Sally and Kelly, 1988; Cox et al., 1992). Auditory cortex was located within two or three slices before and after the crossing of the corpus collossum. The medial geniculate nucleus is visible and contained within the same plane, and can be used as a marker of the correct anteroposterior level. The paired pathway was always from an electrode placed laterally in L-II/III (horizontal stimulation). An unpaired pathway was also present, to insure pathway specificity of the induction protocol and control for nonspecific changes in recording conditions. For the unpaired pathway, the stimulating electrode was placed either on the opposite side of paired pathway or vertically below the recording electrode in the L-VI/white matter border. No significant differences were observed in the paired-pulse ratios between these two sites.

Hippocampal experiments. The hippocampus was dissected out, and transverse hippocampal slices were cut with a vibratome. Intracellular recordings were made from CA1 pyramidal cells. For stimulation, bipolar electrodes were placed in the stratum radiatum near the CA3-CA1 border and at the subicular end of the stratum radiatum.

Recording and stimulation. Intracellular recordings were made with sharp electrodes with an impedance of $40-100 \mathrm{M} \Omega$ when filled with $3 \mathrm{M}$ KAc. All experiments were done under current clamp. Data were sampled and recorded at $10 \mathrm{kHz}$. For hippocampal cells, penetrations were considered acceptable if the resting potential was less than $-60 \mathrm{mV}$, the input resistance was $30 \mathrm{M} \Omega$ or greater, and there were overshooting action potentials. The same criteria were used for neocortical neurons, except that the resting potential criterion was $-70 \mathrm{mV}$. Stainless steel bipolar electrodes were used for stimulation. We favored placing the stimulating electrodes far from the cell being recorded, in order to use higher current intensities for stimulation $(30-800 \mu \mathrm{A}$, duration of $0.1 \mathrm{msec}$ ), since we have observed that during paired-pulse stimulation lower intensities are more likely to result in differential recruitment of axonal fibers.
Protocol. In both hippocampal and neocortical experiments, three different patterns of stimulation were used: paired-pulse stimulation at 50 and $100 \mathrm{msec}$ intervals and a train of 10 pulses at $40 \mathrm{~Hz}$. Each pattern was applied in the above sequence with an intertrial interval of 20 or $30 \mathrm{sec}$ (the unpaired pathway was stimulated $10-15 \mathrm{sec}$ out of phase with the paired pathway). In some neocortical experiments, only paired-pulse stimulation was used (intervals of 50, 100, 200, 300, and $400 \mathrm{msec}$ ). For the induction of LTP, postsynaptic depolarization $(100-150 \mathrm{msec})$ was applied in conjunction with the $40 \mathrm{~Hz}$ train. The degree of depolarization was adjusted to elicit $\sim 10$ spikes during the $100 \mathrm{msec}$ depolarization (generally requiring 2-4 nA). In cortical experiments, pairing was matched with the last five or six pulses, and in the hippocampus depolarization was paired with either the first ("early pairing") or last five pulses ("late pairing"). During training the intertrial interval remained the same ( 20 or $30 \mathrm{sec})$ as during testing. However, since it was previously reported (Colino et al., 1992; my personal observation) that it was difficult to induce LTP with a intertrial interval $>20 \mathrm{sec}$, in most of the hippocampal experiments the intertrial interval was decreased to 10 or 15 sec during training.

Data analysis. The responses to the $40 \mathrm{~Hz}$ train were analyzed before and after the induction of LTP by first averaging traces from a cell during a 6 min window before the beginning of the induction protocol and during a 6 min window (24-30 min) after the end of the induction protocol. In general, each average consisted of four traces (total of 12 trials in which each of three stimulus protocols was applied in alteration). For analysis of the average data, each trace was averaged with the traces from other experiments, thus allowing the visualization of the mean (and SEM) postsynaptic response to a $40 \mathrm{~Hz}$ train from all cells. For calculation of the paired-pulse ratios, the slope or amplitude of the second pulse was determined after the subtraction of the first EPSP. Note that for the paired-pulse plasticity analysis displayed in Figure 4, the number of cells analyzed for the paired and unpaired pathway is different. Although both pathways were always present, in some instances it was not possible to obtain reliable slope measurements of the second EPSP because of polysynaptic contamination; that is, when there was not a clear EPSP onset that was stimulus locked.

\section{RESULTS}

It was first established that L-II/III auditory cortex neurons exhibit associative LTP. Figure $2 A$ shows an example from a single experiment in which a stimulus train $(10$ pulses at $40 \mathrm{~Hz})$ was used to examine STP before and after the induction of LTP. LTP was induced by pairing the second half of the train with postsynaptic depolarization. Pairing occurred at the same interval as testing, every $30 \mathrm{sec}$. Because the first EPSPs were not paired with depolarization, it is possible to follow the induction of LTP during the $10 \mathrm{~min}$ protocol. Note that during induction the potentiation is first observed in the first EPSP, and the later EPSPs exhibit little potentiation, even though these are the ones explicitly being paired with depolarization. The time-series plot shows that LTP lasted at least $30 \mathrm{~min}$ and was specific to the paired pathway. Figure $2 B$ shows the average LTP (as measured by the first EPSP) from 11 experiments.

Figure 3 shows the average interaction between LTP and STP in L-II/III pyramidal neurons. Traces represent the average postsynaptic responses from seven different experiments. In the average traces it can be seen that the potentiation seemed to be larger for the first EPSP. By scaling the first EPSP of the baseline trace to the first EPSP of the posttest trace, it is possible to see that the amplitude of the second to fifth EPSPs is bigger during baseline, indicating relatively less potentiation of the latter EPSPs. The subtraction of the baseline from the posttest trace also reveals a larger degree of absolute change in the first peak. Note that in the scaled traces, the last three EPSPs overlap with the baseline. The subtracted traces show that although these individual EPSPs underwent little potentiation, there was a small increase in a slower DC component. Because all studies were done 
A.
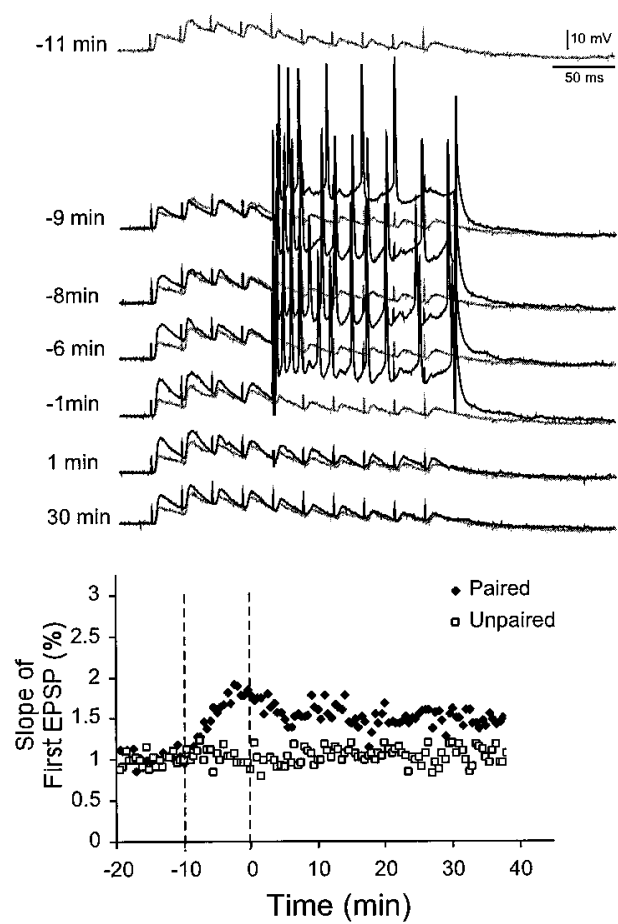

B.

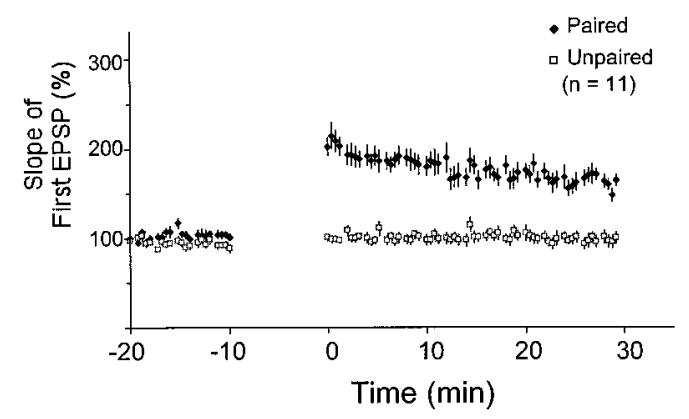

Figure 2. Induction of LTP in a L-II/III pyramidal neuron. $A$, Example of the induction of LTP by pairing depolarization with presynaptic activity. The top trace shows the postsynaptic response to 10 pulses (40 $\mathrm{Hz}$ ) before training $(-11 \mathrm{~min})$. Subsequent traces show the responses during and after induction of LTP. During the induction protocol, depolarization was paired with the latter half of the $40 \mathrm{~Hz}$ train. The baseline trace is repeated for comparison at each point (gray). The bottom panel shows the slope of the first EPSP during the paired and unpaired pathway. $B$, Average LTP from 11 experiments in L-II/III pyramidal neurons.

under intact pharmacology, it is possible that this steady state increase was caused by a decrease in inhibition.

To determine the time window in which the interaction between short- and long-term plasticity is observed, we examined STP plasticity using paired pulses of 50, 100, 200, and $300 \mathrm{msec}$. Figure $4 A$ shows the responses of a single cell to paired pulse before and after the induction of LTP. Figure $4 B$ shows the average paired-pulse plasticity before and after LTP. As expected both paired-pulse facilitation (PPF) and paired-pulse depression (PPD) were observed in L-II/III neurons. On average, PPF was observed at $50 \mathrm{msec}$, and PPD was observed at longer intervals. After the induction of LTP, the small degree of PPF observed at 50 msec was decreased to the extent of becoming PPD. Similarly for the $100 \mathrm{msec}$ interval, there was a decrease in the paired-pulse ratio. In contrast, at intervals of 200 and $300 \mathrm{msec}$, no significant changes in paired-pulse plasticity were observed. Figure 4, $C$ and $D$, shows that no changes were observed for the control pathway.
A.
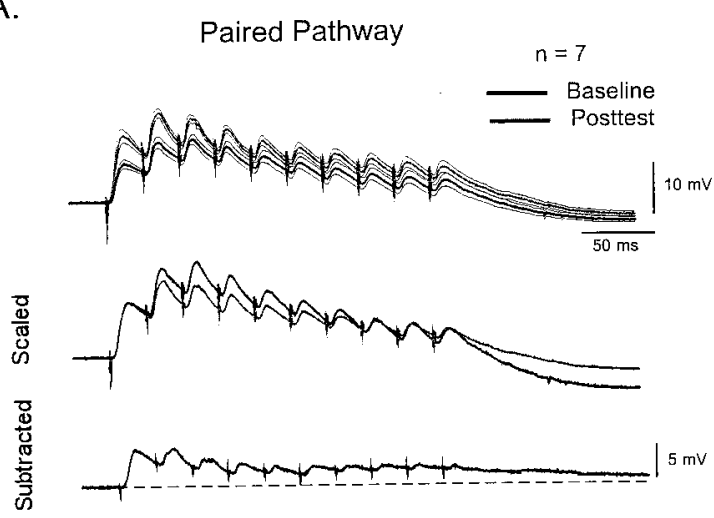

B.

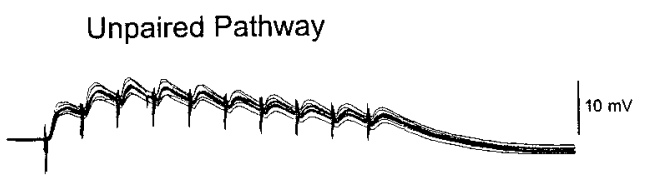

Figure 3. Interaction of LTP and STP in L-II/III neurons. $A$, Each trace represents the average response of seven neurons to the $40 \mathrm{~Hz}$ train before (black) and after (gray) the induction of LTP. The thin lines represent the SEM of the average trace. The middle traces are the same as those displayed above after scaling the first EPSP of the baseline response to the first EPSP of the posttest response. Note that for the second to fifth EPSPs the baseline responses are larger, indicating relatively less potentiation of these EPSPs. The bottom trace shows the subtraction of the baseline from the posttest trace. $B$, Average postsynaptic responses to the unpaired pathway in the same seven cells.

There was no significant difference of the baseline paired-pulse ratios between the paired and unpaired pathways. The pairedpulse results emphasize that changes in inhibition are unlikely to be contributing to the observed interaction between LTP and STP. The small increase in the steady state ("DC component") to the train could be in part caused by a decrease in the strength of fast or slow IPSPs. However, to produce and apparent decrease in EPSP amplitude of the latter pulses, an increase in inhibition would be necessary. Furthermore, at an interval of $100 \mathrm{msec}$, it is unlikely that the fast IPSP would effect the slope of EPSPs.

The results presented above clearly show that LTP produces changes in STP in L-II/III pyramidal neurons. To determine if this is a general feature of associative LTP, we next performed similar experiments in hippocampal CA1 neurons. Figure $5 A$ shows an example of a single experiment in which paired-pulse stimulation (50 and $100 \mathrm{msec}$ ) and a $40 \mathrm{~Hz}$ train were used to characterize STP before and after the induction of LTP. Note that in contrast to the LTP observed in L-II/III neurons there was clearly a large degree of potentiation of the EPSPs occurring later in the train. Figure $5 B$ shows the average LTP of the first EPSP, averaged over eight different cells. The average degree of potentiation was $180 \pm 16 \%$, as compared to the neocortex, in which average potentiation was $158 \pm 7 \%$. Figure $5 \mathrm{C}$ shows that on average there was no change in PPF as the result of the induction of LTP.

LTP was induced in CA1 neurons using two different protocols. Postsynaptic depolarization was applied in conjunction with the first (early pairing) or second half (late pairing) of the $40 \mathrm{~Hz}$ train. When using extracellular stimulation to elicit a train of EPSPs, it is possible that changes in the threshold for eliciting action potentials in axonal fibers occur. In this scenario, STP 


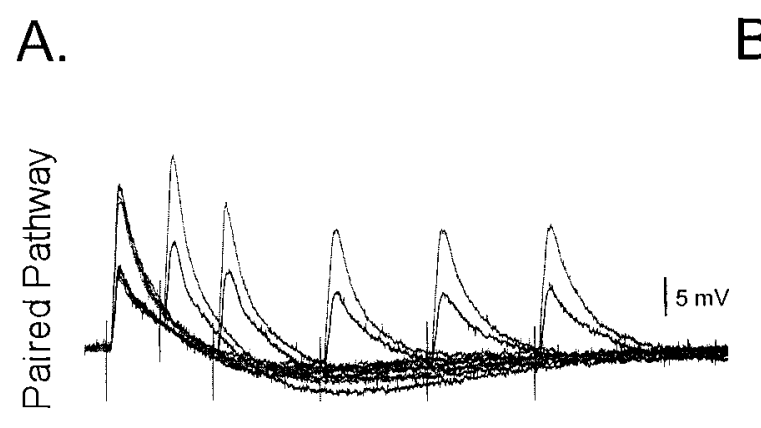

B.
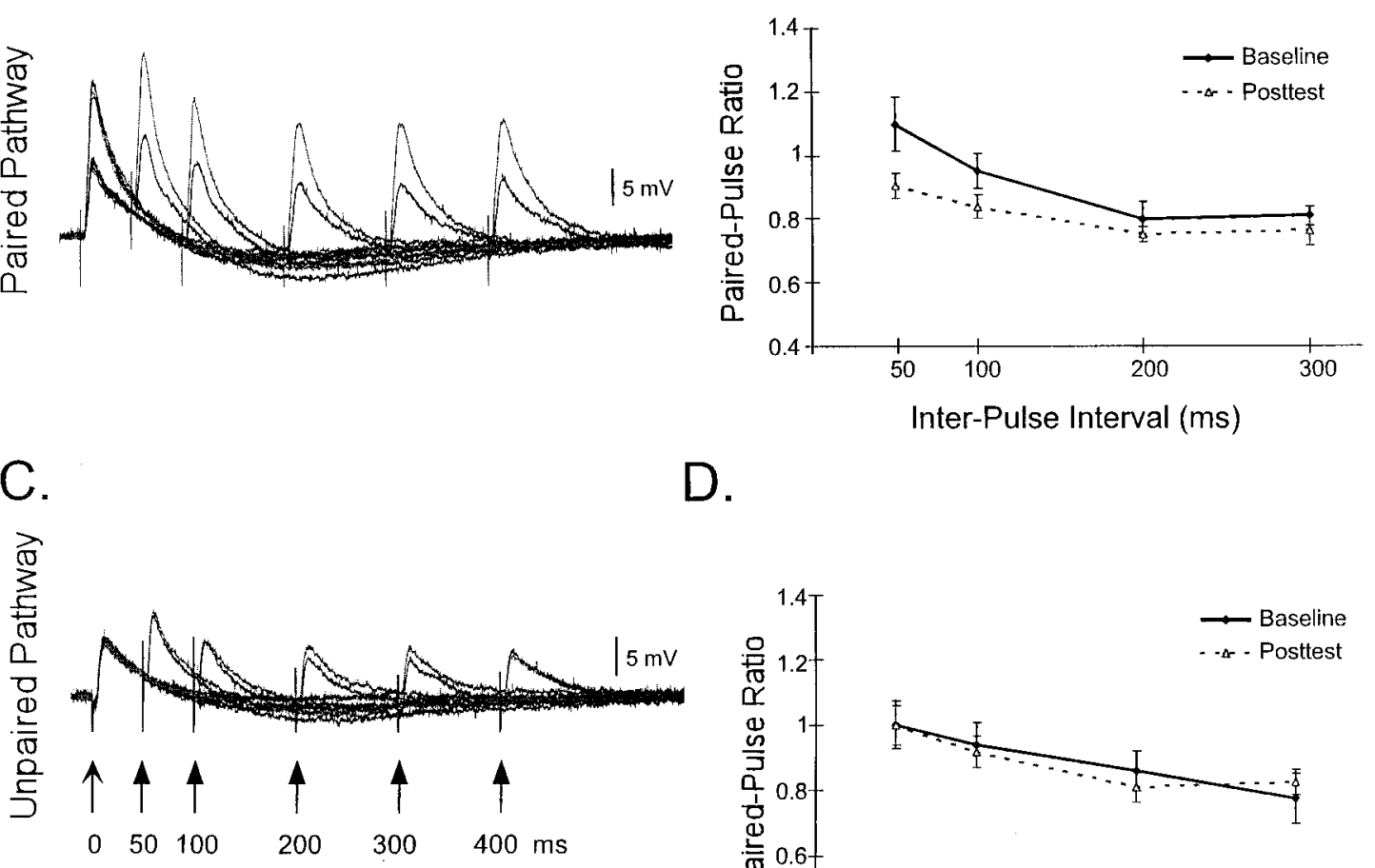

D.

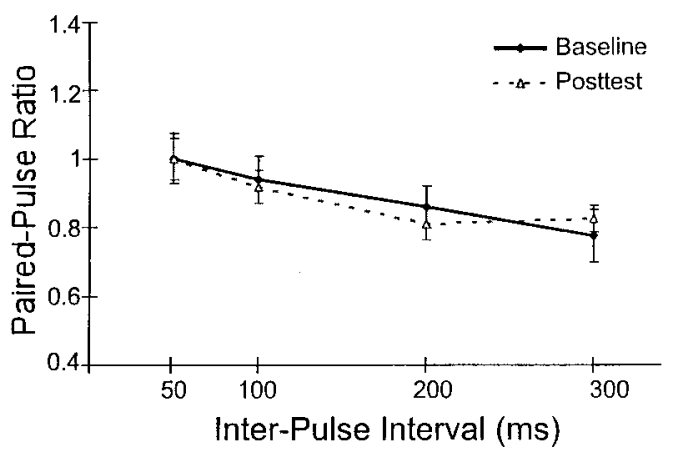

Figure 4. LTP modifies paired-pulse plasticity in L-II/III neurons. Traces from a single experiment before (black) and after ( gray) the induction of LTP in the paired $(A)$ and unpaired $(C)$ pathways. Right panels, Average paired-pulse ratio (slope of the second pulse/slope of first pulse) before and after the induction of LTP for the paired $(B)$ and unpaired $(D)$ pathways. In $B$, each point represents an average of $15,15,6$, and 6 cells for the four different intervals (in ascending order). The values of 50 and $100 \mathrm{msec}$ are significantly different before and after LTP $(p<0.02)$. In $D$, the number of cells for each point was $12,12,8$, and 8 . Note that in $A$ the apparent facilitation at $50 \mathrm{msec}$ is mostly temporal summation. The paired-pulse ratios shown in $B$ and $D$ are calculated from the slopes of the second EPSP after subtraction of the first EPSP.

could be contaminated by recruitment artifacts: apparent facilitation could be caused in part by additional axonal recruitment throughout the train. By depolarizing the postsynaptic cell at different times, it is possible to detect if any recruitment artifacts were consistently occurring in these experiments. For example, if additional axons are being recruited late in the train, they would undergo LTP with the late but not the early protocol. Thus, different results would be obtained when comparing data from the early and late protocol. Figure $6 A$ shows that were no consistent differences in the effect of LTP on STP using the two different protocols (scaled data not shown). Figure $6 B$ shows the pooled average traces from all eight cells. In contrast to what was seen for the L-II/III neurons, it was clear that potentiation appeared to be uniform throughout the duration of the train. This is confirmed by scaling the first EPSP of the baseline trace to that of the posttest. Scaling reveals an almost complete overlap of all the remaining EPSPs. The subtraction trace also reveals a clear potentiation of the later EPSPs, and that this potentiation was a result of an increase in the amplitude of each EPSP. Figure $6 C$ shows that no significant changes in LTP or STP were observed in the unpaired pathway.

\section{DISCUSSION}

The results presented here show that the same pairing protocol induces LTP in both CA1 and L-II/III pyramidal neurons. How- ever, LTP differs in relation to its effect on STP, and thus is likely to differ in its functional consequences on neural processing. In the hippocampus, LTP corresponds to changing the synaptic weight or gain by a given constant: synaptic efficacy is enhanced to the same degree irrespective of the recent activity in that synapse. In contrast, in L-II/III neurons LTP did not reflect a constant change in the strength of a synapse: potentiation was dependent on the recent history of activity of that synapse. These results are the first results showing an interaction between LTP and STP in L-II/III cortical neurons. However, they are consistent with previous results in $\mathrm{L}-\mathrm{V}$ pyramidal neurons, which report that relatively little or no potentiation of EPSPs occur later in a train, an effect termed redistribution of synaptic strength (Markram and Tsodyks, 1996). Our hippocampal results are consistent with a recent field potential (Pananceau et al., 1998) and a whole-cell study (Selig et al., 1999) that also used brief trains of stimulation to analyze the interaction between LTP and STP. Together these data suggest that the LTP may be significantly different in the hippocampus and neocortex, regarding its effects on STP, and thus on temporal selectivity. In order to understand the functional role of neocortical LTP, it will be important to determine whether the results observed in L-II/III and in L-V hold true at all neocortical synapses. LTP at the thalamocorticalL-IV synapse has been described and is known to be associative 
A.

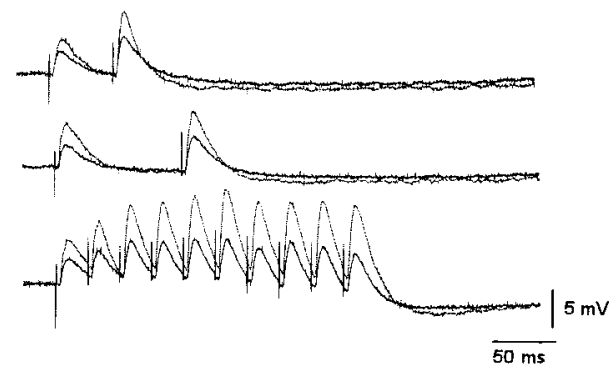

B.

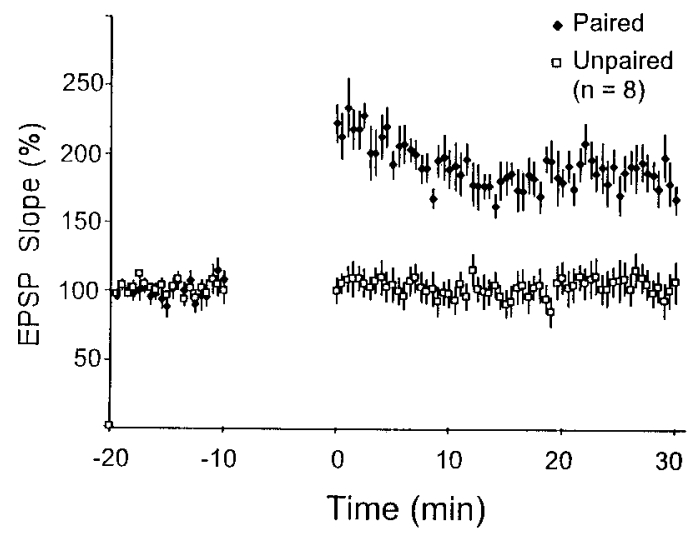

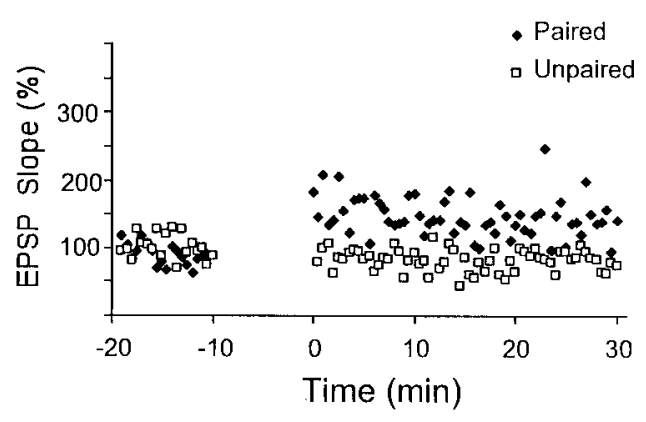

C.

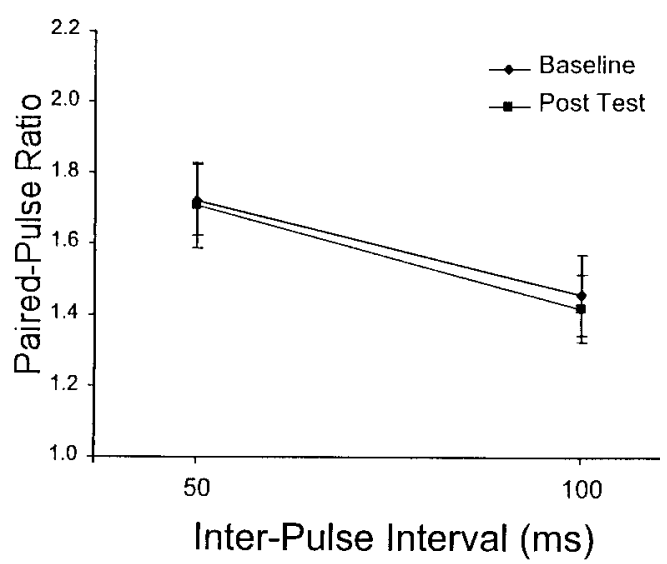

Figure 5. A, LTP in hippocampal CA1 pyramidal neurons. Traces of a single experiment showing the responses to paired-pulse stimulation at intervals of 50 and $100 \mathrm{msec}$, and a $40 \mathrm{~Hz}$ train before (black) and after LTP (gray). Note that the differences in the amplitude of the first EPSP in the three different conditions reflect the normal variability of synaptic amplitude. The slope of the first EPSP is shown throughout the experiment for the paired and unpaired pathway on the right. $B$, Average LTP for all eight cells. $C$, Average paired-pulse ratio for the same eight cells. There was no significant change in the paired-pulse ratio after the induction of LTP for either the 50 or $100 \mathrm{msec}$ interval.

and NMDA-dependent (Crair and Malenka, 1995). However, it is not yet known if LTP at these synapses is "CA1-like" or "L-II/ III like". In the hippocampus, a clear interaction between LTP and STP is also observed in the hippocampus at the mossyfiber-CA3 synapses (Zalutsky and Nicoll, 1990). However, LTP at these synapses is generally not considered to be associative or Hebbian in nature. Additionally, unlike CA1 and neocortical LTP (Kirkwood et al., 1993), mossy-fiber-CA3 LTP is not NMDA-dependent.

\section{Short-term plasticity}

It is important to note that there were baseline differences in STP observed at L-II/III and CA1 neurons. In agreement with previous reports, facilitation was observed in the Schaffer collateralCA1 synapses (Creager et al., 1980; Manabe et al., 1993; Buonomano and Merzenich, 1996). Both facilitation and depression is observed in neocortical synapses onto excitatory neurons (Thomson and Deuchars, 1994; Markram and Tsodyks, 1996; Ramoa and Sur, 1996; Stratford et al., 1996; Gil et al., 1997; Buonomano and Merzenich, 1998b; Reyes and Sakmann, 1999). However, depression seems to predominate, and facilitation to the degree observed in CA3 is rare. Stratford et al. (1996) have shown that part of this diversity is determined by which neocortical synapses are involved. Reyes and Sakmann (1999) have shown that there is also likely to be developmental changes occurring in STP. Specifically, synapses between L-II/III pyramidal neurons exhibited PPD early in development and PPF later in development.

It is unlikely that the differences in initial STP accounted for the observed differences between hippocampus and neocortex. Often it was the L-II/III neurons that initially exhibited the highest degree of short-term facilitation that exhibited the largest LTP-induced changes in STP. Furthermore, recent experiments in CA1 neurons have used pharmacological or stimulus manipulations to decrease the initial level of facilitation, to better resemble the baseline condition observed in the neocortex (Pananceau et al., 1998; Selig et al., 1999). Under such conditions, uniform LTP is still observed across the whole stimulus train. It seems likely that neocortical synapses may exhibit more short-term depression, precisely because LTP induced previously during development resulted in preferential potentiation of early EPSPs, thus producing short-term depression.

The effects of LTP on STP have generally been analyzed using paired-pulse stimulation. Using this technique, most reports in CA1 synapses report that, on average, LTP does not effect pairedpulse plasticity (Muller and Lynch, 1988; Manabe et al., 1993; Schulz et al., 1994), however some reports indicate that LTP produces a decrease in PPF or a change that correlates with initial levels of PPF (Kuhnt and Voronin, 1988; Schulz et al., 1994; Wang and Kelly, 1997). It seems likely that brief trains of pulses would provide a more sensitive measure of the interaction between LTP and STP, however the current study and two others have not revealed such an interaction (Pananceau et al., 1998; Selig et al., 1999). It is possible that differences in the induction protocols have contributed to some of the experimental discrepancies. In the current study, it is of interest that depolarization during the early or late pulses did not produce different results, 
A.

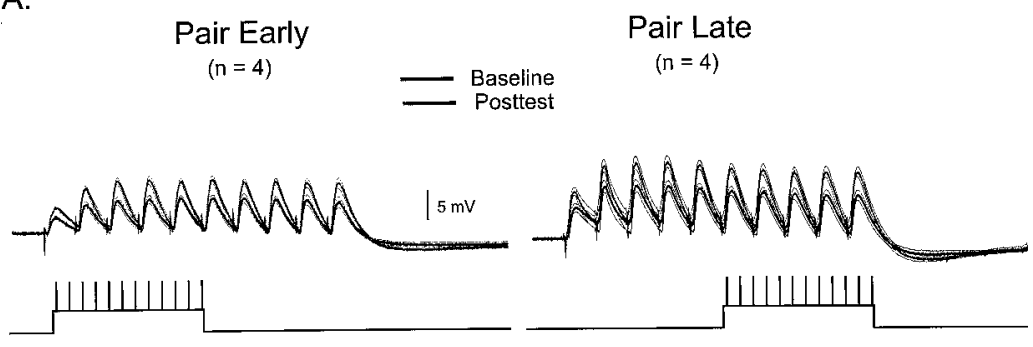

B.

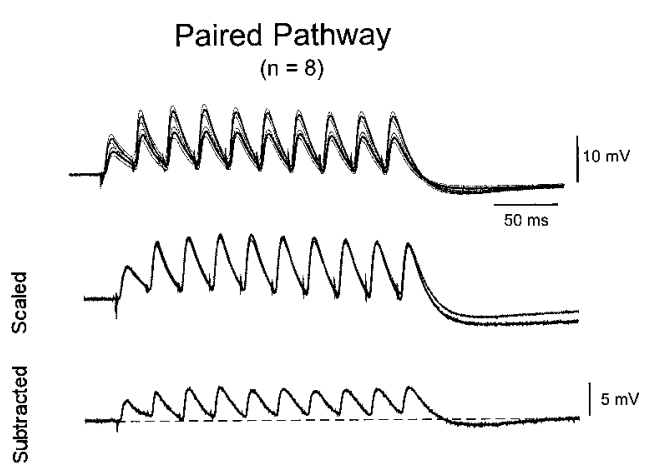

C.

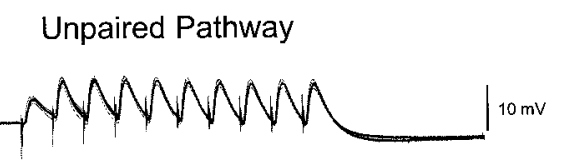

Figure 6. In the hippocampus, LTP uniformly potentiates all EPSPs of a train. $A$, Two different protocols were used to induce LTP. Depolarization was paired either with the first five pulses (early) or last five pulses (late). Each trace shows the average of four separate experiments before (black) and after (gray) the induction of LTP. Thin lines represent the SEM. In both groups, potentiation was uniform across the length of the train. B, Top traces represent the average baseline and posttest trace from all eight cells. Middle traces are the same as those shown above after scaling. Note that the overlap of the normalized traces indicate potentiation was the same across all EPSPs. Bottom trace shows the subtraction of the baseline from the posttest traces. $C$, In the unpaired pathway there were no significant changes before and after the induction of LTP. suggesting that short-term facilitation is not itself modulated in a training-sensitive manner.

\section{Mechanistic implications}

The mechanisms underlying short-term synaptic plasticity are generally assumed to be presynaptic. Thus, LTP-induced changes in STP have been used to argue for a presynaptic site of expression for LTP (Kuhnt and Voronin, 1988; Schulz et al., 1994). A few recent reports have put into question the notion that STP is a purely presynaptic phenomenon (Clark et al., 1994; Wang and Kelly, 1996). Given our incomplete understanding of the neural mechanisms underlying STP, it is difficult to use the interaction between LTP and STP as evidence in favor or against the involvement of a presynaptic site for the expression of LTP. In the current experiments, it was observed that the same protocol, which is assumed to rely on postsynaptic induction, does or does not alter STP, depending on the synapses being studied. These results emphasize the fact that biochemical mechanisms underlying LTP must not only regulate the efficacy of synapses to isolated EPSPs, but that specialized biochemical mechanisms are likely to be in place to modulate short-term plasticity, whether these are presynaptic or postsynaptic. A surprisingly large number of biochemical pathways, particularly different kinases, have been implicated in LTP. The reason why so many different pathways are involved with increases in synaptic strength has remained unclear. One possibility is that such complexity is required to maintain or alter STP in parallel with the expression and maintenance of LTP.

\section{Functional implications}

The differential effect of LTP on STP, as well as differences in the initial degree of short-term plasticity suggests that STP may have multiple functional roles. Indeed, various nonmutually exclusive hypotheses have been made regarding the role of STP in information processing. One hypothesis is that short-term changes in synaptic efficacy underlie temporal processing. Specifically, shortterm changes in synaptic efficacy produce time-dependent changes in the state of local networks, which in turn result in distinct population response to different temporal stimuli (Buonomano and Merzenich, 1995; Buonomano et al., 1997). It has also been suggested that STP may provide a mechanism for "on-line" modulation in certain types of behaviors (Fisher et al., 1997). Others have hypothesized that short-term depression between excitatory cortical neurons may play a role in gain control (Abbott et al., 1997) or maintaining the stability of cortical circuits by keeping positive feedback in check (Galarreta and Hestrin, 1998). Our results indicate that because in the hippocampus LTP did not alter the temporal profile of the postsynaptic response, LTP may be involved in forms of learning in which preservation of temporal information is important. In contrast, potentiation of cortical synapses results in amplification of the onset responses, resulting in changes in the temporal selectivity or gain control of neurons. It will be of interest to determine whether LTD produces a converse phenomenon, increasing the probability of neurons responding to later input events. In layer II/III pyramidal neurons, the paired-pulse study revealed that the interaction between short- and long-term plasticity is limited to intervals $<100-200 \mathrm{msec}$. Thus, long-term potentiation would not be expected to alter the response profile to temporal stimuli $<5-10 \mathrm{~Hz}$. This observation may reflect the segmentation time, the interval over which incoming signals are treated as independent, rather than different components of the same stimulus. 
LTP of the excitatory synapses between L-II/III pyramidal neurons have been proposed to underlie some forms of experience-dependent learning (Armstrong-James et al., 1994; Diamond et al., 1994). Specifically, whisker pairing (in which all but two vibrissae of a pad are removed), results in L-II/III pyramidal neurons in the barrel cortex that exhibit multi-vibrissae receptive fields. It has been suggested that these new receptive fields emerge as a result of increased lateral flow of information among supragranular pyramidal neurons as a result of LTP. If the observations made here also apply to barrel cortex, a prediction that emerges is that in vivo whisker pairing will result in increased responses to the adjacent vibrissae, however, that a sequence of whisker stimulation should reveal that the increase is limited to the first few stimuli. Thus, in considering the functional role of LTP in learning and memory it is necessary to take into account the effects of LTP on short-term plasticity and whether these effects are consistent with in vivo and behavioral data.

\section{REFERENCES}

Abbott LF, Varela JA, Kamal S, Nelson SB (1997) Synaptic depression and cortical gain control. Science 275:220-224.

Armstrong-James M, Diamond M, Ebner FF (1994) An innocuous bias in whisker use in adult rats modifies receptive fields of barrel cortex neurons. J Neurosci 14:6978-6991.

Buonomano DV, Merzenich MM (1995) Temporal information transformed into a spatial code by a neural network with realistic properties. Science 267:1028-1030.

Buonomano DV, Merzenich MM (1996) Associative synaptic plasticity in hippocampal CA1 neurons is not sensitive to unpaired presynaptic activity. J Neurophysiol 76:631-636.

Buonomano DV, Merzenich MM (1998a) Cortical plasticity: from synapses to maps. Annu Rev Neurosci 21:149-186.

Buonomano DV, Merzenich MM (1998b) Net interaction between different forms of short-term synaptic plasticity and slow-IPSPs in the hippocampus and auditory cortex. J Neurophysiol 80:1765-1774.

Buonomano DV, Hickmott PW, Merzenich MM (1997) Temporal to spatial transformations and context-sensitive plasticity in hippocampal slices. Proc Natl Acad Sci USA 94:10403-10408.

Clark KA, Randall AD, Collingridge GL (1994) A comparison of paired-pulse facilitation of AMPA and NMDA receptor-mediated excitatory postsynaptic currents in the hippocampus. Exp Brain Res 101:272-278.

Colino A, Huang Y-Y, Malenka RC (1992) Characterization of the integration time for the stabilization of long-term potentiation in area CA1 of the hippocampus. J Neurosci 12:180-187.

Cox CL, Metherate R, Weinberger NM, Ashe A (1992) Synaptic potentials and effects of amino acid antagonists in auditory cortex. Brain Res Bull 28:401-410.

Crair MC, Malenka RC, (1995) A critical period for long-term potentiation at thalamocortical synapses. Nature 375:325-328.

Creager R, Dunwiddie T, Lynch G (1980) Paired-pulse and frequency facilitation in the CA1 region of the in vitro hippocampus. J Physiol (Lond) 299:409-424.

Diamond ME, Huang W, Ebner F (1994) Laminar comparison of somatosensory cortical plasticity. Science 265:1885-1888.
Fisher SA, Fischer SA, Carew TJ (1997) Multiple overlapping processes underlying short-term synaptic enhancement. Trends Neurosci 20:170-177.

Galarreta M, Hestrin, S (1998) Frequency-dependent synaptic depression and the balance of excitation and inhibition in the neocortex. Nature Neurosci 1:587-594.

Gil Z, Connors BW, Amitai Y (1997) Differential regulation of neocortical synapses by neuromodulators and activity. Neuron 19:679-686.

Kirkwood A, Dudek SM, Gold JT, Aizenman CD, Bear MF (1993) Common forms of synaptic plasticity in the hippocampus and neocortex in vitro. Science 260:1518-1521.

Kuhnt U, Voronin LL (1988) Interaction between paired-pulse facilitation and long-term potentiation in area CA1 of guinea-pig hippocampal slices: application of quantal analysis. Neuroscience 62:391-397.

Manabe T, Wyllie DJA, Perkel DJ, Nicoll RA (1993) Modulation of synaptic transmission and long-term potentiation: effects on paired pulse facilitation and EPSP variance in the CA1 region of the hippocampus. J Neurophysiol 70:1451-1459.

Markram H, Tsodyks M (1996) Redistribution of synaptic efficacy between neocortical pyramidal neurons. Nature 382:807-810.

Muller D, Lynch G (1988) Long-term potentiation differentially affects two components of synaptic responses in hippocampus. Proc Natl Acad Sci USA 85:9346-9350.

Pananceau M, Chen H-X, Gustafsson B (1998) Short-term facilitation evoked during brief afferent tetani is not altered by long-term potentiation in the guinea-pig hippocampal CA1 region. J Physiol (Lond) 508:503-514.

Ramoa AS, Sur M (1996) Short-term synaptic plasticity in the visual cortex during development. Cereb Cortex 6:640-646.

Reyes A, Sakmann B (1999) Developmental switch in the short-term modification of unitary EPSPs evoked in layer II/III and layer 5 pyramidal neurons of rat neocortex. J Neurosci 19:3827-3835.

Sally S, Kelly JB (1988) Organization of auditory cortex in the albino rat: sound frequency. J Neurophysiol 59:1627-1638.

Schulz PE, Cook EP, Johnston D (1994) Changes in paired-pulse facilitation suggest presynaptic involvement in long-term potentiation. J Neurosci 14:5325-5337.

Selig DK, Nicoll RA, Malenka RC (1999) Hippocampal long-term potentiation preserves the fidelity of postsynaptic responses to presynaptic bursts. J Neurosci 19:1236-1246.

Stratford KJ, Tarczy-Hornoch K, Martin KAC, Bannister NJ, Jack JJB (1996) Excitatory synaptic inputs to spiky stellate cells in cat visual cortex. Nature 382:258-261.

Thomson AM, Deuchars J (1994) Temporal and spatial properties of local circuits in neocortex. Trends Neurosci 17:120-126.

Wang J-H, Kelly PT (1996) Regulation of synaptic facilitation by postsynaptic $\mathrm{Ca}^{2+} / \mathrm{CaM}$ pathways in hippocampal CA1 neurons. J Neurophysiol 76:276-285.

Wang J-H, Kelly PT (1997) Attenuation of paired-pulse facilitation associated with synaptic potentiation mediated by postsynaptic mechanisms. J Neurophysiol 78:2707-2716.

Winer JA, Larue DT (1987) Patterns of reciprocity in auditory thalamocortical and corticothalamic connections: study with horseradish peroxidase and autoradiographic methods in the rat medial geniculate body. J Comp Neurol 257:282-315.

Zalutsky RA, Nicoll RA (1990) Comparison of two forms of long-term potentiation in single hippocampal neurons. Science 248:1619-1624. 